\title{
Theoretical description of drawing body shape in an inclined seam with longwall top coal caving mining
}

\author{
Jiachen Wang ${ }^{1,2} \cdot$ Weijie Wei ${ }^{1,2}$ (D) Jinwang Zhang ${ }^{1,2}$
}

Received: 13 May 2019/Revised: 29 September 2019/Accepted: 29 November 2019/Published online: 11 December 2019

(C) The Author(s) 2019

\begin{abstract}
Understanding the characteristics of drawing body shape is essential for optimization of drawing parameters in longwall top coal caving mining. In this study, both physical experiments and theoretical analysis are employed to investigate these characteristics and derive a theoretical equation for the drawing body shape along the working face in an inclined seam. By analyzing the initial positions of drawn marked particles, the characteristics of the drawing body shape for different seam dip angles are obtained. It is shown that the drawing body of the top coal exhibits a shape-difference and volume-symmetry characteristic, on taking a vertical line through the center of support opening as the axis of symmetry, the shapes of the drawing body on the two sides of this axis are clearly different, but their volumes are equal. By establishing theoretical models of the drawing body in the initial drawing stage and the normal drawing stage, a theoretical equation for the drawing body in an inclined seam is proposed, which can accurately describe the characteristics of the drawing body shape. The shape characteristics and volume symmetry of the drawing body are further analyzed by comparing the results of theoretical calculations and numerical simulations. It is shown that one side of the drawing body is divided into two parts by an inflection point, with the lower part being a variation development area. This variation development area increases gradually with increasing seam dip angle, resulting in an asymmetry of the drawing body shape. However, the volume symmetry coefficient fluctuates around 1 for all values of the seam dip angle variation, and the volumes of the drawing body on the two sides are more or less equal as the variation development volume is more or less equal to the cut volume. Both theoretical calculations and numerical simulations confirm that the drawing body of the top coal exhibits the shape-difference and volume-symmetry characteristic.
\end{abstract}

Keywords Longwall top coal caving mining · Inclined seam · Top coal $\cdot$ Drawing body shape $\cdot$ Equation for drawing body

\section{Introduction}

Longwall top coal caving (LTCC) mining is an efficient method for extracting thick coal seams, and, with the development of high-performance equipment, it has become widely used in inclined and steeply inclined seams.

Weijie Wei

wilson1998@foxmail.com

1 School of Energy and Mining Engineering, China University of Mining and Technology (Beijing), Beijing 100083, China

2 Coal Industry Engineering Research Center of Top-coal Caving Mining, Beijing 100083, China
Most current research on LTCC in inclined seams concerns the top coal drawing mechanism, support stability, failure characteristics of overburden strata, and control of surrounding rock. Wang and Zhang (2015), Wang et al. (2019), Zhang et al. (2018a, b), and Song and Konietzky (2019) used the boundary-body-ratio (BBR) system, based on a series of laboratory experiments, field measurements and numerical calculations, to reveal the relationships among the drawing body and boundary of top coal and the recovery ratio in the LTCC panel. Yasitli and Unver (2005) and Unver and Yasitli (2006) studied the shape characteristics of the boundary and drawing body, and proposed that the uniformity of fracture of the top coal is directly related 
to the fluidity of top coal caving. To make the control of the surrounding rock more effective and improve the stability of the supports of the LTCC panel in steeply inclined coal seams, Wang et al. (2017) and Li et al. (2017) analyzed the forces imposed on the supports and proposed a new caving method drawing downward segment by segment while drawing upward in each segment. Based on a large number of creep experiments, Verma and Mishra (2015) investigated the time-dependent deformation behavior of immediate coal measures rock and explained the effect of timedependent deformation on roof stability. Le et al. $(2018,2019)$ studied the stress distribution, coal and rock failures, and roof strata rupture in the LTCC panel, and then analyzed the impact of overburden movement on top coal caving. Jeromel et al. (2010) and Likar et al. (2012) investigated the multilevel LTCC process by FLAC $^{3 \mathrm{D}}$ and in situ measurements during coal excavation in the Velenje Coal Mine. Kumar et al. (2015) revealed that a weak coal mass and a high vertical stress on the coal seam were the two most important geological parameters determining the success of LTCC, and this method has been used for bordand-pillar mining in India. Vakili and Hebblewhite (2010) developed a method for assessing cavability through the study of six factors that can affect the caving of top coal and thereby increased overall understanding of the caving mechanism in LTCC. However, there has been little research on the shape characteristics or on deriving a theoretical equation for the drawing body in an inclined seam, which would provide the basis for developing a drawing method to improve top coal recovery. Melo et al. (2007, 2009) derived an equation for the drawing body in a flat seam in the cases of both single and multiple openings, based on the Bergmark-Roos and kinematic models. Yu et al. (2017) used stochastic medium theory to obtain an equation for the drawing body shape and the boundary of the top coal. Taking account of the influence of the shield beam on the drawing process, Wang et al. (2016) derived an equation for the drawing body along the direction of advance of the working face and found that the drawing body was a cut-variation ellipsoid, whose lower part was cut by the shield beam and whose upper part developed toward the front of the support.

In summary, the drawing body shape has been studied under the condition of a flat seam and along the direction of advance of the working face, with the aim of providing help in choosing an appropriate drawing interval. However, in an inclined seam, the drawing body shape along the working face is affected by the seam dip angle, and this will directly influence the determination of the drawing parameters and the ability to improve top coal recovery. To date, there have been no theoretical studies of the drawing body in an inclined seam. Therefore, in this paper, to remedy this deficit, an equation for the drawing body along the working face in an inclined seam is derived for the first time, and the characteristics of the drawing body shape during both the initial and normal drawing stages are analyzed in detail.

\section{Physical experiments}

\subsection{Experimental device and model layout}

To study the influence of the seam dip angle $\alpha$ on drawing body shape, drawing experiments were carried out using a custom-built experimental device. The geometric similarity ratio of the experimental model was 30:1, the coal seam was laid $300 \mathrm{~mm}$ thick with cyan limestone particles (with the heights of the bottom and top coal being $100 \mathrm{~mm}$ and $200 \mathrm{~mm}$, respectively), and the rock was laid $200 \mathrm{~mm}$ thick with white limestone particles. As shown in Fig. 1, the supports were numbered 1-24 from left to right. Six supports at each end of the device were in a no-drawing area to eliminate boundary effects on the model during the top coal drawing process. In addition, the marked particles laid over supports Nos. 4-21 in the coal seam were arranged in seven layers, with 54 (3 rows and 18 columns) in each layer, and the space between the layers in the vertical direction was $30 \mathrm{~mm}$.

Initial models of top coal drawing experiments with six values of seam dip angle $\left(\alpha=0^{\circ}, 10^{\circ}, 20^{\circ}, 30^{\circ}, 40^{\circ}\right.$, and $50^{\circ}$ ) were established to study the influence of this angle on the drawing body shape of the top coal. Figure 2 shows three of the models at the initial stage, in which the drawing sequence of the top coal is from support No. 7 to support No. 18. After each drawing, the mass of drawn top coal and the number of drawn marked particles were recorded to analyze the shape of drawing body of the top coal.

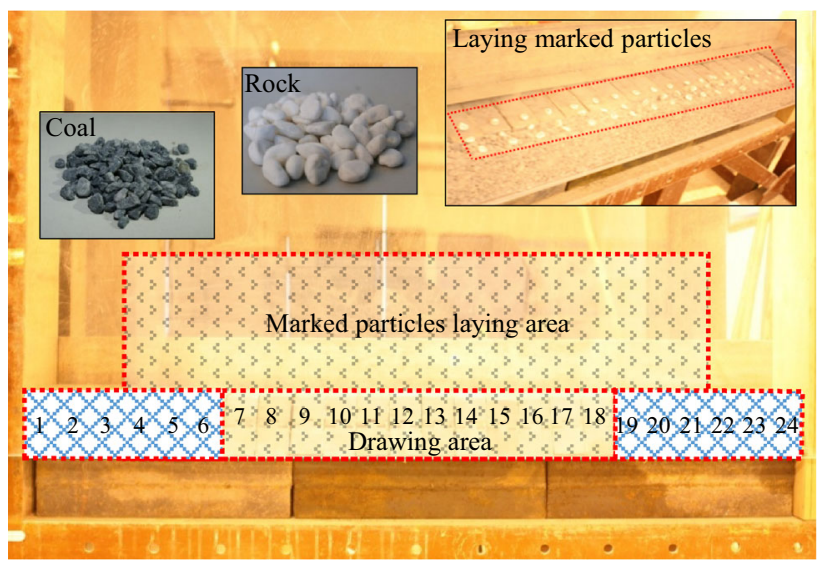

Fig. 1 Experimental device and layout of marker particles 

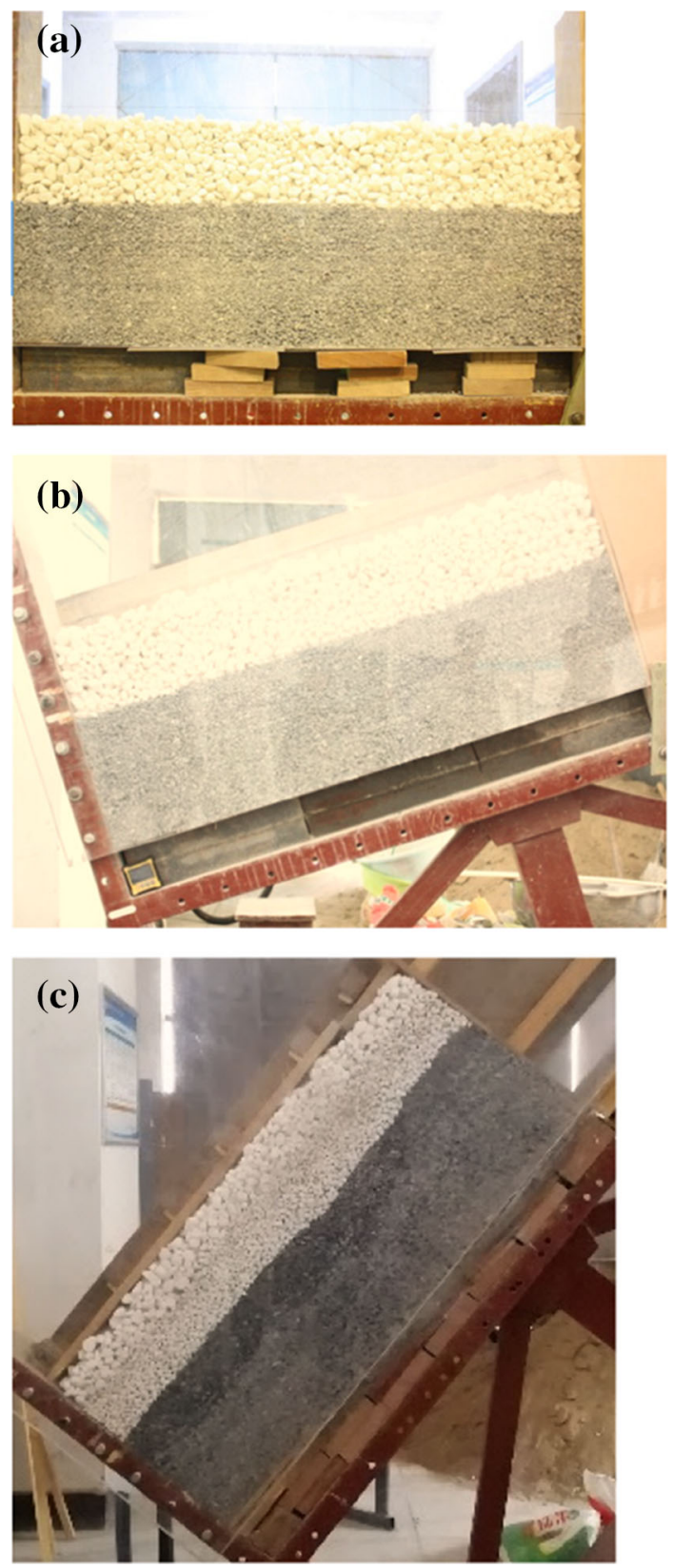

Fig. 2 Three of the experimental models in the initial stage: $\mathbf{a} \alpha=0^{\circ}$; b $\alpha=20^{\circ} ; \mathbf{c} \alpha=50$

\subsection{Experimental results and analysis}

Figure 3 shows the curves of variation of the mass of drawn top coal $m_{c}$ with support number and of the total mass of drawn top coal $m_{c t}$ with seam dip angle. It can be seen that with increasing seam dip angle, the initial $m_{c}\left(m_{c}\right.$ from support No. 7) increased gradually. However, the difference between adjacent drawings also increased significantly, which is not conducive to a balanced top coal drawing process. From Fig. $3 b$, we can see that $m_{c t}$ also increased gradually with increasing seam dip angle. At the
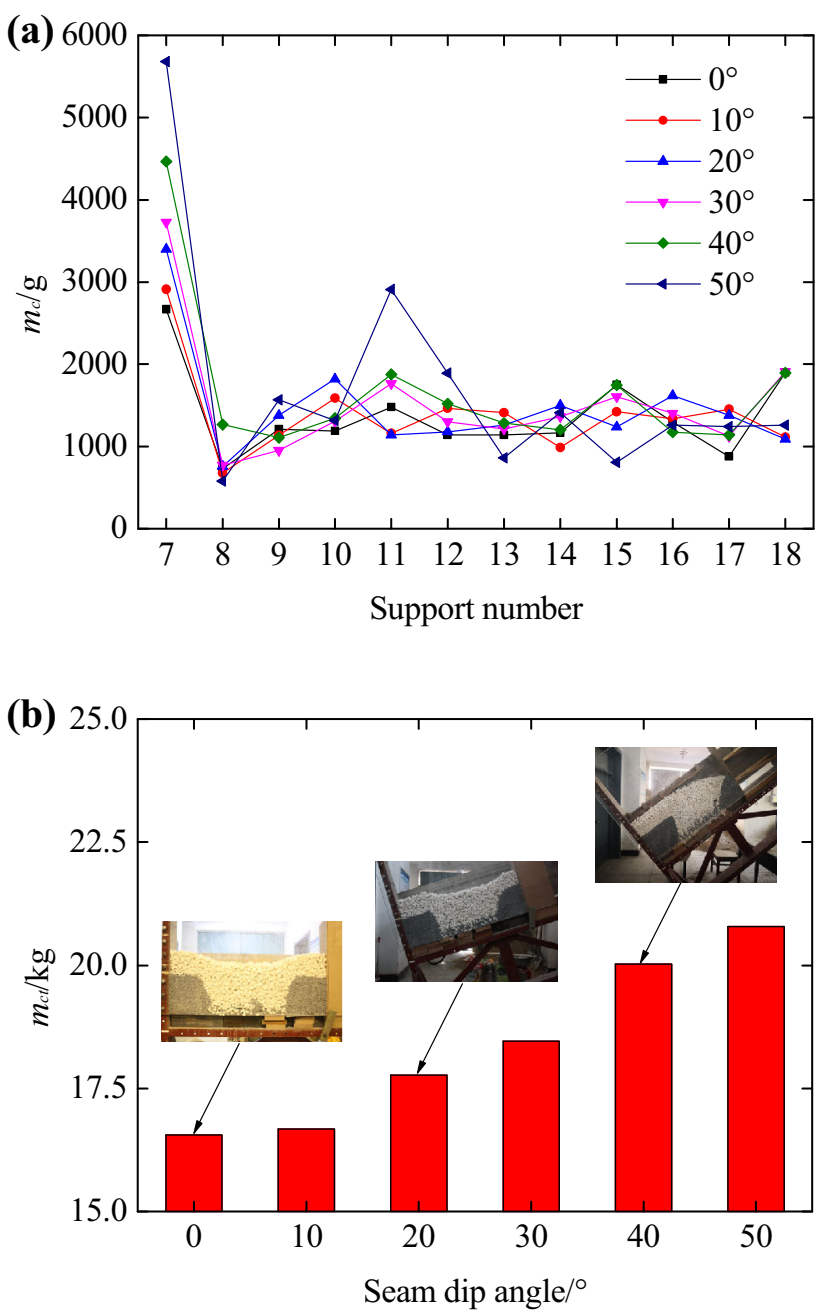

Fig. 3 a Variation of mass of drawn top coal with support number; b variation of total mass of drawn top coal with seam dip angle

same time, from the shape of the boundary of the top coal, it can be seen that $m_{c}$ at the lower end of the panel decreased gradually, while $m_{c}$ at the upper end of the panel increased significantly, which does not help to improve top coal recovery at the lower end or the stability of the roadway at the upper end. To solve these problems, it is helpful to derive a theoretical equation for the drawing body in an inclined seam and determine its shape characteristics.

Figure 4 shows the drawing body shapes in the initial drawing stage (support No. 7) for different seam dip angles. The red dots are the drawn marked particles from support No. 7, while the black dots are the marked particles that are not drawn. The red dotted line is the vertical line through the center of the support opening.

It can be seen from Fig. 4 that when $\alpha=0^{\circ}$, the drawn marked particles included all marked particles above support No. 7 and most of marked particles above supports No. 6 and No. 8. Taking the red dotted line in Fig. 4 as the axis 

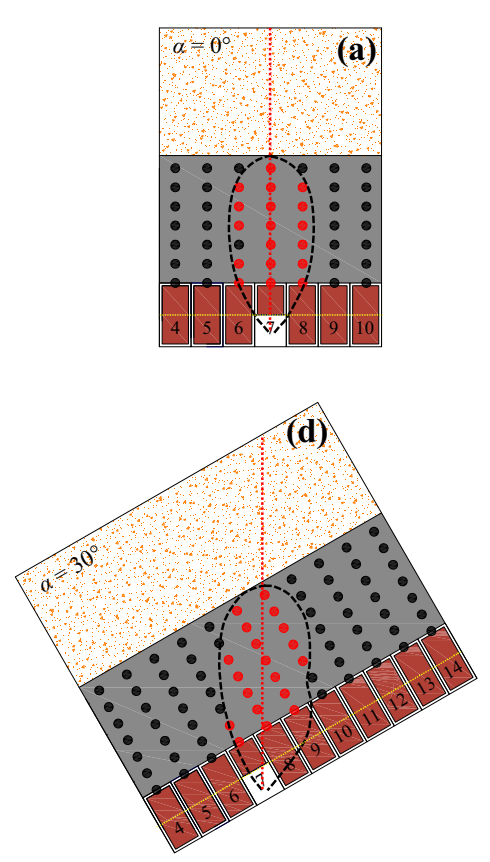
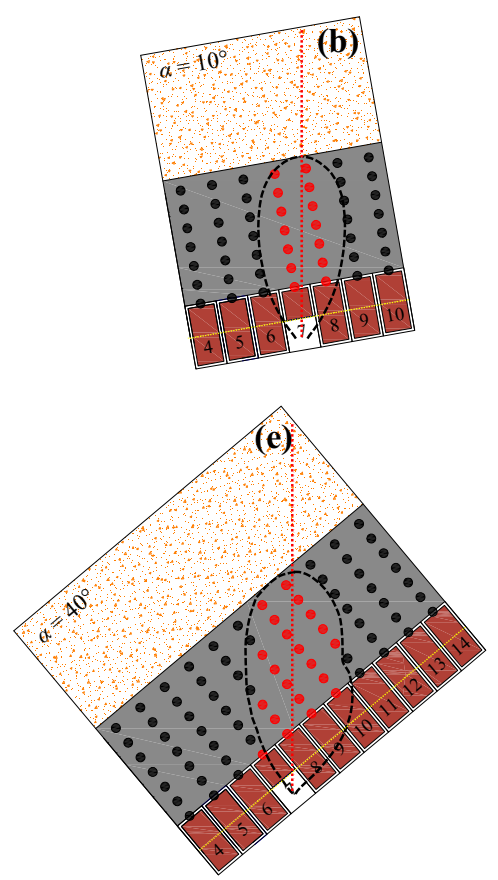

(c)
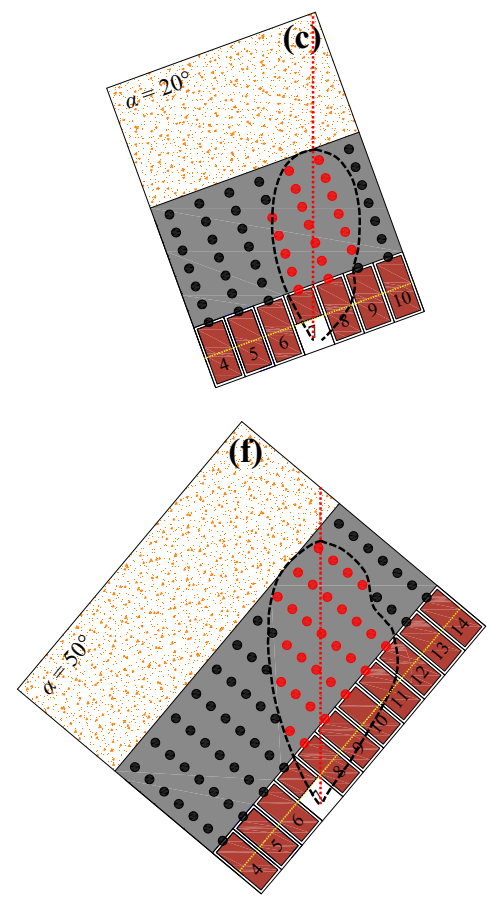

Fig. 4 Inversion of the drawing body shape in the initial drawing stage: $\mathbf{a} \alpha=0^{\circ} ; \mathbf{b} \alpha=10^{\circ} ; \mathbf{c} \alpha=20^{\circ} ; \mathbf{d} \alpha=30^{\circ} ; \mathbf{e} \alpha=40^{\circ} ; \mathbf{f} \alpha=50^{\circ}$

of symmetry, the drawing body shape was basically symmetrical, with its width on each side being equal to the width of a support. However, for an inclined seam, the drawing body developed rapidly on approaching the upper side of the panel, the range of drawn marked particles on the lower right side was clearly wider than that on the lower left side, and the shapes of the drawing body on the two sides were clearly different. Furthermore, this difference in shape was greater for larger seam dip angles.

The number of drawn marked particles on each side in the initial drawing stage was counted and is plotted in
Fig. 5 (the particles on the axis of symmetry are not included on either of the sides). The values represented by the blue and red columns are the numbers of drawn marked particles on the left and right sides, respectively. It can be seen from Fig. 5 that the number of drawn marked particles on the left side was basically equal to that on the right side, which indicates that the volumes of the drawing body on the two sides were also basically equal for different seam dip angles.

The experimental results showed that the influence of the seam dip angle on the drawing body is manifested only

(a) Number of marked particles on the left

(b) Number of marked particles on the right

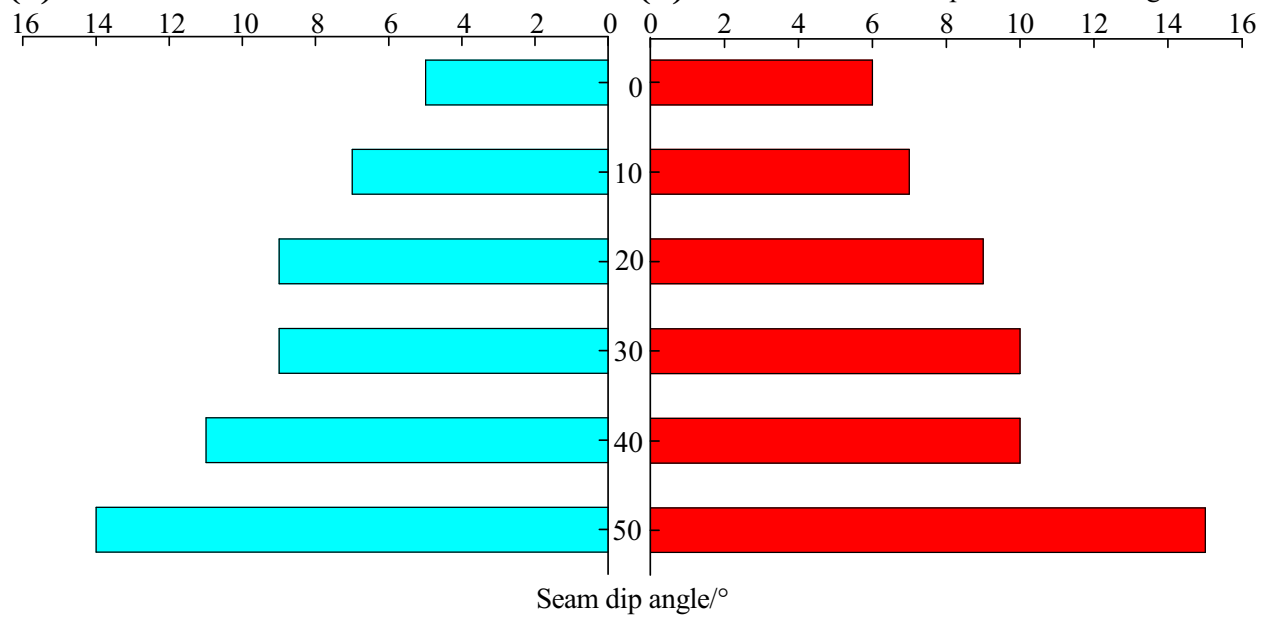

Fig. 5 Numbers of drawn marked particles on $\mathbf{a}$ the left side and $\mathbf{b}$ the right side in the initial drawing stage 
in its shape, not in its volume. Thus, the drawing body in an inclined seam is characterized by shape difference and volume symmetry. The difference in drawing body shape between the two sides is caused by the nonzero seam dip angle. With increasing angle, the difference in shape becomes greater, but the volume symmetry of the two sides is unaffected.

\section{Theoretical equation for drawing body shape in an inclined seam}

The overall shape of the drawing body in Fig. 4 is similar to that obtained from the Bergmark-Roos (B-R) model (Melo et al. 2007, 2009), and so a modification of this model is proposed in this paper for describing the drawing body shape in an inclined seam. For the top coal drawing process in an inclined seam, the following factors need to be taken into account: (1) the inclination of the support opening; (2) the different boundary conditions on the two ends of the opening, with an infinite boundary condition on the lower end and an inclined boundary condition on the upper end of opening; (3) the smaller value of the friction between particles and supports at the upper end of the opening compared with that among top coal particles at the lower end, which is beneficial for top coal drawing at the upper end. These factors differ from those in a flat seam, so it can be expected that the drawing body shape will have different characteristics in an inclined seam. The equation for the drawing body along the working face is derived here for both the initial and normal drawing stages.

\subsection{Equation for the drawing body in the initial drawing stage}

\subsubsection{Theoretical model of drawing body}

As shown in Fig. 6, polar coordinates with $O$ as origin are established, with the drawing area including the angular

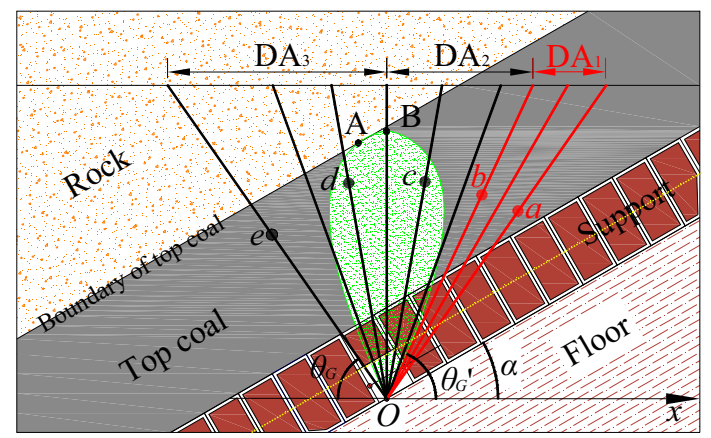

Fig. 6 Theoretical model of the drawing body in the initial drawing stage in an inclined seam range from $\theta_{G}$ to $180^{\circ}-\theta_{G}$, which is divided into three areas according to the motion of the top coal particles. In Fig. 6, the first drawing area $\mathrm{DA}_{1}$ contains the angular range from $\theta_{G}$ to $\theta_{G}^{\prime}$, the second drawing area $\mathrm{DA}_{2}$ contains the range from $\theta_{G}^{\prime}$ to $90^{\circ}$, and the third drawing area $\mathrm{DA}_{3}$ contains the range from $90^{\circ}$ to $180^{\circ}-\theta_{G}$. Here, $\theta_{G}$ is the maximum angle of particle motion, $\theta_{G}^{\prime}$ is the angle at which the particles move along a straight line and just past the upper boundary of the support opening, and $O$ is the center point of the lines tracing the motion of the particles.

When the top coal particles in $\mathrm{DA}_{1}$ (particles $a$ and $b$ in Fig. 6) move in a straight line, they will hit the tail beam of the support. Furthermore, if the particle motion follows the principle that the particles flow toward the opening along paths of minimum resistance, then the paths traced by the top coal particles in $\mathrm{DA}_{1}$ will change under the constraint imposed by the tail beam. Therefore, $\mathrm{DA}_{1}$ is also called the trace-variation zone. The friction between particles and tail beam is smaller than that among top coal particles, which results in a rapid flow of top coal particles at the upper end of the opening.

For top coal particles in $\mathrm{DA}_{2}$ and $\mathrm{DA}_{3}$ (particles $c, d$, and $e$ in Fig. 6), the paths traced by their motion are not affected by the tail beam, in contrast to particles in $\mathrm{DA}_{1}$. To simplify the following calculation, we assume that the motion of particles in $\mathrm{DA}_{2}$ and $\mathrm{DA}_{3}$ is not affected by the seam dip angle and that they still flow along straight lines toward $O$.

Furthermore, the first drawn rock particle is theoretically located at the tangent point (point $A$ in Fig. 6) between the drawing body and the boundary of the top coal. In contrast with the case of a flat coal seam, the highest point (point $B$ in Fig. 6) of the drawing body is slightly lower than the boundary of the top coal.

Figure 7 shows the distribution of the velocity field along the working face in $\mathrm{PFC}^{3 \mathrm{D}}$. From Fig. 7a, it can be seen that when $\alpha=0^{\circ}$, the velocity field distribution on the two sides of the opening is symmetrical. The velocity is small before the particles reach the opening, but it suddenly increases after the particles have passed through the opening. From Fig. 7b, it can be seen that the velocity field on the two sides of the opening is no longer symmetrical in an inclined seam, and the particle velocity at the upper end is significantly larger than that at the lower end. At the same time, the particles are no longer moving toward $O$ in a certain range near the tail beam (the trace-variation zone), as a consequence of which the paths traced by the top coal particles change. Therefore, it can be considered that in the trace-variation zone, the center point of the lines traced by particle movement is no longer $O$, but rather the point $O^{\prime}$ at the upper boundary of the opening, as shown in Fig. 8. 

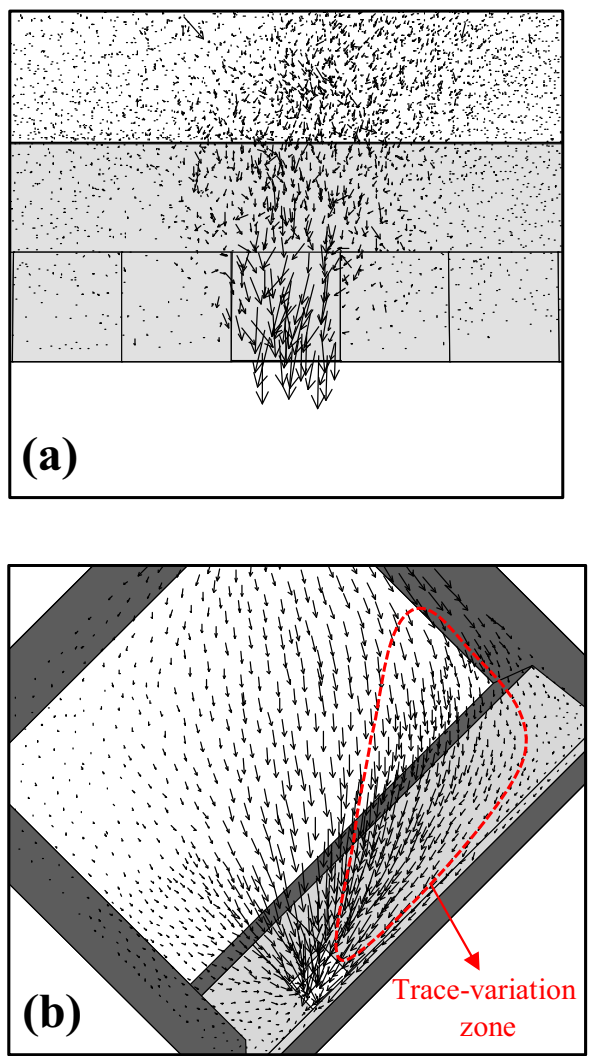

Fig. 7 Distribution of the particle velocity field along the working face: a flat seam; $\mathbf{b}$ inclined seam

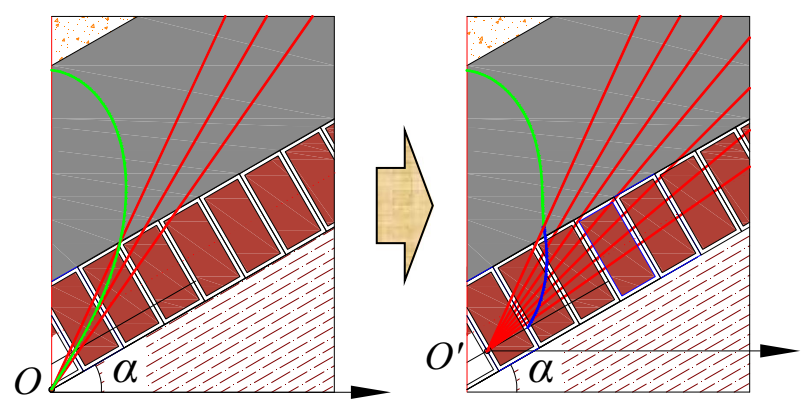

Fig. 8 Changes in the lines tracing particle motion in the tracevariation zone

Based on the above analysis, the variation in the drawing body shape caused by the changes in velocity of the particles and the lines traced by their motion is shown in Fig. 9. It can be seen that the lower right part of the drawing body is larger than the lower left part, and the drawing body develops rapidly toward the upper side of the panel face, as shown by the cross-hatched area in Fig. 9 (the variation development area), which increases with increasing seam dip angle.

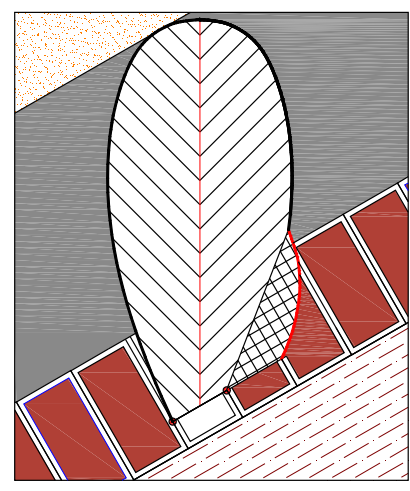

Fig. 9 Variation of drawing body shape in an inclined seam

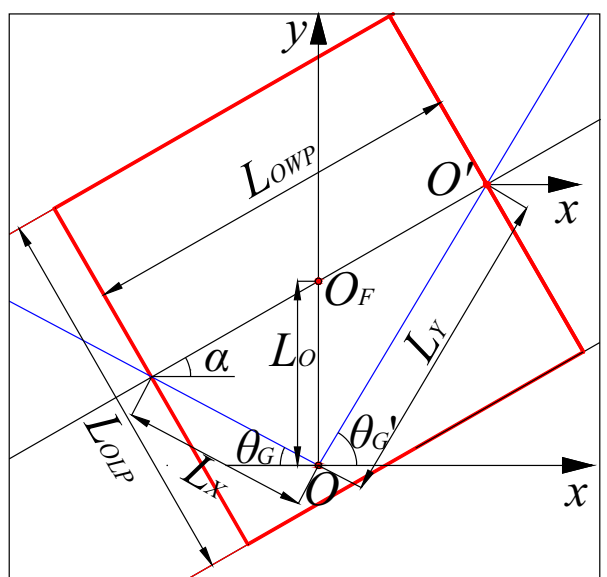

Fig. 10 Projection of the support opening on the vertical plane

\subsubsection{Equation for drawing body}

We consider the support opening, shown by the red box in Fig. 10 as a projection on the vertical plane. A rectangular coordinate system is established by taking the point $O$ as the origin, the vertical line through $O$ as the $y$ axis and the horizontal line through point $O$ as the $x$ axis. A polar coordinate system is also established with $O$ as the origin and with the horizontal line through $O$ as the polar axis. In addition, the point $O_{F}$ is the center of the opening, $L_{O W P}$ is the projected width of the opening, $L_{O L P}$ is the projected length of opening, and $L_{X}$ is the distance between the point at the lower boundary of the opening and $O$.

According to the law of sines, $\theta_{G}^{\prime}$ satisfies

$$
\left\{\begin{array}{l}
\frac{\frac{L_{O W P}}{2}}{\sin \left(\frac{\pi}{2}-\theta_{G}\right)}=\frac{L_{X}}{\sin \left(\frac{\pi}{2}-\alpha\right)} \\
\frac{L_{X}}{\sin \left(\theta_{G}^{\prime}-\alpha\right)}=\frac{L_{O W P}}{\sin \left(\pi-\theta_{G}-\theta_{G}^{\prime}\right)}
\end{array}\right.
$$

from which the following relation among $\theta_{G}^{\prime}, \theta_{G}$, and $\alpha$ can be obtained: 
$\tan \theta_{\mathrm{G}}^{\prime}=\tan \theta_{G}+2 \tan \alpha$

Therefore, $\theta_{G}^{\prime}$ is given by.

$\theta_{G}^{\prime}=\arctan \left(\tan \theta_{G}+2 \tan \alpha\right)$

When $\alpha \neq 0$, it is obvious from Eq. (3) that $\theta_{G}^{\prime}$ is always larger than $\theta_{G}$. That is, in an inclined seam, there will always exist a region $\mathrm{DA}_{1}$ where particles cannot flow along straight lines toward $O$. The angle $\theta_{G}$ depends on the internal friction angle $\varphi_{0}$ of the particles:

$\theta_{G}=45^{\circ}+\frac{\varphi_{0}}{2}$

$\theta_{G}^{\prime}$ can be obtained by combining Eqs. (3) and (4), and then the ranges of $\mathrm{DA}_{1}, \mathrm{DA}_{2}$, and $\mathrm{DA}_{3}$ can be determined.

The derivation of an equation for the drawing body in an inclined seam can be divided into two parts.

(1) The motion of the top coal particles in $\mathrm{DA}_{2}$ and $\mathrm{DA}_{3}$ can be considered as straight-line motion toward the point $O$, satisfying the following equation:

$\rho(\theta)=\frac{1}{2}\left(g \sin \theta-g \sin \theta_{G}\right) t^{2}$

In an inclined coal seam, the distance $h_{m v}$ between the seam roof and the point $O_{F}$ and the distance $L_{O}$ between the points $O$ and $O_{F}$ can be obtained as

$h_{m v}=\frac{h_{m}}{\cos \alpha}-\frac{L_{O L P}}{2 \cos \alpha}=\frac{2 h_{m}-L_{O L} \sin \beta}{2 \cos \alpha}$

$L_{O}=\frac{L_{O W P} \sin \left(\alpha+\theta_{G}\right)}{2 \cos \theta_{G}}=\frac{L_{O W} \sin \left(\alpha+\theta_{G}\right)}{2 \cos \theta_{G}}$

where $h_{m}$ is the thickness of the seam, $L_{O L}$ is the inclined length of the opening, $L_{O W}$ is the width of the opening, and $\beta$ is the angle between the tail beam and the seam floor.

The linear equation for the seam roof $l_{0}$ in rectangular coordinates is

$$
\begin{aligned}
y & =\tan \alpha \cdot x+\frac{2 h_{m}-L_{O L} \sin \beta}{2 \cos \alpha}+\frac{L_{O W} \sin \left(\alpha+\theta_{G}\right)}{2 \cos \theta_{G}} \\
& =\tan \alpha \cdot x+L_{C}
\end{aligned}
$$

where $L_{C}$ is the distance between the seam roof and the point $O$. The equation for $l_{0}$ in polar coordinates is

$\rho(\theta)=\frac{L_{C}}{\sin \theta-\tan \alpha \cos \theta}$

Theoretically, the drawing process follows the principle of the drawing opening being closed when the first rock particle is drawn out. The polar coordinates of the tangent point $A$ are $\left(\theta_{A}, \rho\left(\theta_{A}\right)\right)$, and those of the highest point $B$ are $\left(\theta_{B}, \rho\left(\theta_{B}\right)\right)$. Because the point $A$ is located on the roof $l_{0}$, its coordinates satisfy Eq. (9), i.e.,

$\rho\left(\theta_{A}\right)=\frac{L_{C}}{\sin \theta_{A}-\tan \alpha \cos \theta_{A}}$
Substitution of Eq. (10) into Eq. (5) gives

$$
\frac{t^{2}}{2}==\frac{\rho\left(\theta_{A}\right)}{g \sin \theta_{A}-g \sin \theta_{G}}
$$

from which the following equation for the drawing body in $\mathrm{DA}_{2}$ and $\mathrm{DA}_{3}\left(l_{2-3}\right)$ is obtained:

$\rho(\theta)=\rho\left(\theta_{A}\right) \frac{\sin \theta-\sin \theta_{G}}{\sin \theta_{A}-\sin \theta_{G}}$

The derivatives of Eq. (9) and Eq. (12) can then be calculated as

$$
\left\{\begin{array}{r}
\rho(\theta)_{l_{0}}^{\prime}=-\frac{L_{C}(\cos \theta+\tan \alpha \sin \theta)}{(\sin \theta-\tan \alpha \cos \theta)^{2}} \\
\rho(\theta)_{l_{2-3}}^{\prime}=\frac{\rho\left(\theta_{A}\right) \cos \theta}{\sin \theta_{A}-\sin \theta_{G}}
\end{array}\right.
$$

The two curves are both tangent to the point $A$, and so their derivatives at $A$ must be equal. From the expressions in Eq. (13) at $\theta=\theta_{A}$, the polar coordinates of $A$ therefore satisfy

$$
\frac{\left(1+\tan \alpha \tan \theta_{A}\right)\left(\sin \theta_{G}-\sin \theta_{A}\right)}{\sin \theta_{A}-\tan \alpha \cos \theta_{A}}=1
$$

This can be solved for $\theta_{A}$, and then the $\rho\left(\theta_{A}\right)$ and the equation for the drawing body in $\mathrm{DA}_{2}$ and $\mathrm{DA}_{3}$ can be obtained. When $\theta=90^{\circ}$, the maximum height of the drawing body is given by

$\rho_{\max }=\rho\left(\theta_{A}\right) \frac{1-\sin \theta_{G}}{\sin \theta_{A}-\sin \theta_{G}}$

(2) For the top coal particles in $\mathrm{DA}_{1}$, the influence of the seam dip angle on the drawing body shape is obvious and the particles in this area flow along straight lines toward $O^{\prime}$. In an inclined seam, the particles of the upper end of the panel face outside the drawing area also flow toward the opening, which indicates that the frictional force acting on the top coal particles in $\mathrm{DA}_{1}$ has changed, let us say to $g f_{1}$. As shown in Fig. 10, the new polar coordinates take the point $O^{\prime}$ as the origin, and the horizontal line through the upper boundary of opening as the polar axis, and the equation for the drawing body in the new polar coordinates $\left(l_{1}\right)$ is now

$\rho\left(\theta_{O^{\prime}}\right)=K_{1} \rho_{\max } \frac{\sin \theta_{O^{\prime}}-f_{1}}{1-\sin \theta_{G}}$

where $\theta_{O^{\prime}}$ is the polar angle of a given point in the new polar coordinates, $K_{1}$ is the correction coefficient for the polar radius, and $f_{1}$ is the correction coefficient for the $\mathrm{DA}_{1}$, which includes the angular range from $\alpha$ to $\theta_{G}^{\prime}$ in the new polar coordinates. For $l_{1}$ and $l_{2-3}$ to connect exactly, the following must hold: 
$\rho_{\max } \frac{\sin \theta_{G}^{\prime}-\sin \theta_{G}}{1-\sin \theta_{G}}-L_{Y}=K_{1} \rho_{\max } \frac{\sin \theta_{G}^{\prime}-f_{1}}{1-\sin \theta_{G}}$

where $L_{Y}$ is the distance between $O^{\prime}$ and point $O$. As can be seen from Fig. 10, $L_{Y}$ can be obtained from

$\frac{L_{Y}}{\sin \left(\alpha+\frac{\pi}{2}\right)}=\frac{\frac{L_{\text {OWP }}}{2}}{\sin \left(\frac{\pi}{2}-\theta_{G}^{\prime}\right)}$

When $\theta_{O^{\prime}}=\alpha$, the polar radius $\rho(\alpha)$ is given by

$\rho(\alpha)=K_{1} \rho_{\max } \frac{\sin \alpha-f_{1}}{1-\sin \theta_{G}}$

Finally, by combining Eqs. (17)-(19), the correction coefficients $f_{1}$ and $K_{1}$ can be obtained as follows:

$$
\left\{\begin{array}{l}
f_{1}=\frac{M \sin \alpha-\sin \theta_{G}^{\prime}}{M-1} \\
K_{1}=\frac{\rho(\alpha)\left(1-\sin \theta_{G}\right)}{\rho_{\max }\left(\sin \alpha-f_{1}\right)} \\
M=\frac{\rho_{\max }\left(\sin \theta_{G}^{\prime}-\sin \theta_{G}\right)}{\rho(\alpha)\left(1-\sin \theta_{G}\right)}-\frac{L_{O W} \cos \alpha}{2 \cos \theta_{G}^{\prime} \rho(\alpha)}
\end{array}\right.
$$

In summary, the equation for the drawing body in an inclined seam in the initial caving stage is as follows:

$\begin{cases}l_{1}: \rho\left(\theta_{O^{\prime}}\right)=\frac{\rho(\alpha)}{\left(\sin \alpha-f_{1}\right)}\left(\sin \theta_{O^{\prime}}-\frac{M \sin \alpha-\sin \theta_{G}^{\prime}}{M-1}\right), & \alpha \leq \theta_{O^{\prime}} \leq \theta_{G}^{\prime} \\ l_{2-3}: \rho(\theta)=\rho\left(\theta_{A}\right) \frac{\sin \theta-\sin \theta_{G}}{\sin \theta_{A}-\sin \theta_{G}}, & \theta_{G}^{\prime} \leq \theta \leq 180^{\circ}-\theta_{G}^{\prime}\end{cases}$

\subsection{Equation for the drawing body in the normal drawing stage}

As shown in Fig. 11, the drawing area is also divided into three parts in the normal drawing stage, and the particle motion in each part is essentially the same as in the initial drawing stage. That is, the line tracing the motion of a particle in $\mathrm{DA}_{1}$ (particle $a$ in Fig. 11) is constrained by the tail beam, and the particles cannot flow toward $O$. Conversely, particles in $\mathrm{DA}_{2}$ and $\mathrm{DA}_{3}$ (particles $b, c$, and $d$ in Fig. 11) still flow normally toward $O$. Therefore, the

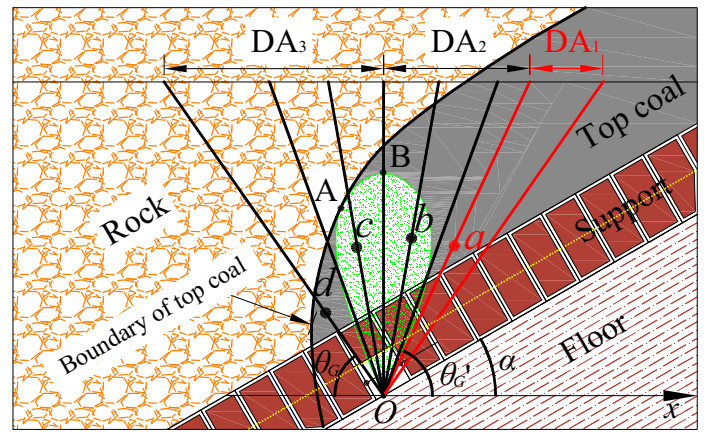

Fig. 11 Theoretical model of drawing body in the normal drawing stage in an inclined seam equation for the drawing body in the normal drawing stage is still given by Eq. (21). The maximum height and volume of the drawing body in the normal drawing stage are smaller than those in the initial drawing stage. This is because the shape of the boundary of the top coal changes from a straight line to a curve in the normal drawing stage, which reduces the actual maximum height of the drawn top coal, and the development of the drawing body is then limited by the boundary of the top coal.

To obtain the equation for the drawing body in the normal drawing stage in an inclined seam, it is necessary to know the polar coordinates of the tangent point $A$. According to Wang and Zhang (2015) and Wei et al. (2018), the boundary of the top coal can be approximately described by a parabolic equation, which we shall assume here takes the form

$y^{2}+C_{1} y+C_{2} x+C_{3}=0$

Then, the polar coordinates of the tangent point $A$ must satisfy

$$
\left\{\begin{array}{l}
\frac{\rho\left(\theta_{A}\right)^{\prime} \tan \theta_{A}+\rho\left(\theta_{A}\right)}{\rho\left(\theta_{A}\right) \tan \theta_{A}-\rho\left(\theta_{A}\right)^{\prime}}=\frac{C_{2}}{2 \rho\left(\theta_{A}\right) \sin \theta_{A}+C_{1}} \\
\rho\left(\theta_{A}\right)^{\prime}=\frac{\rho\left(\theta_{A}\right) \cos \theta}{\sin \theta_{A}-\sin \theta_{G}}
\end{array}\right.
$$

The polar coordinates of $A$ can be obtained from Eq. (23) or by reversing the initial coordinates of the first drawn rock particle in a numerical simulation. We denote the correction coefficients by $f_{2}$ and $K_{2}$, which are again given by Eq. (20). The equation for the drawing body in the normal drawing stage in an inclined seam is then as follows:

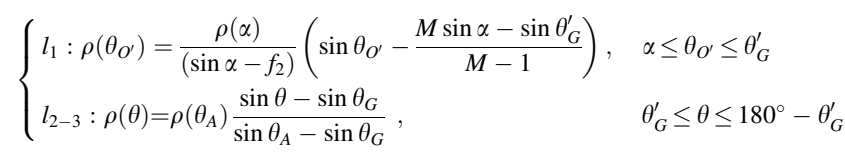

\section{Shape-difference and volume-symmetry characteristic}

The equations for the drawing body in the initial and normal drawing stages are shown in Eqs. (21) and (24), respectively. To explain and verify theoretically the shapedifference and volume-symmetry characteristic of the drawing body found in top coal drawing experiments, the shape characteristics and volume symmetry of the drawing body are analyzed by comparing the results of theoretical calculations and numerical simulations. 


\subsection{Shape difference of drawing body}

\subsubsection{Initial drawing stage}

Using the values of the basic numerical simulation parameters from Zhang et al. (2018a), namely, $h_{m}=9 \mathrm{~m}$, $L_{O L}=1.7 \mathrm{~m}, L_{O W}=1.5 \mathrm{~m}, \beta=60^{\circ}$, and $\theta_{G}=50^{\circ}$, the parameters $\theta_{G}^{\prime}, L_{O}, L_{C}, \theta_{A}, \rho\left(\theta_{A}\right), \rho_{\max }, K_{1}$, and $f_{1}$ are calculated for different seam dip angles, and the results are shown in Table 1. The equation for the drawing body in the initial drawing stage is then solved with these parameter values to give the theoretical drawing body shapes (the red full curve in Fig. 12). Comparison with the numerical simulation results (the blue dashed curve in Fig. 12) shows a good match, with the small difference possibly being due to the use of spherical elements to simulate the top coal particles in $\mathrm{PFC}^{3 \mathrm{D}}$. The right side of the drawing body is divided into two parts by an inflection point (the small yellow circle in Fig. 12), with the upper part being more or less symmetrical in shape with the left side of the drawing body, while the lower part develops rapidly toward the upper end of the panel face, corresponding to the variation development area shown cross-hatched in Fig. 9. In addition, the angular coordinate of the inflection point is the same according to both the theoretical equation and the numerical simulation, which further verifies the correctness of the equation.

The variations of the drawing body parameters with increasing seam dip angle in the initial drawing stage are shown in Fig. 13. It can be seen that with increasing seam dip angle, both $\theta_{A}$ and $\theta_{G}^{\prime}$ gradually increase, which indicates that the position of the tangent point $A$ gradually falls on the left side of the drawing body, while the position of the inflection point gradually rises on the right side. In general, the influence of the seam dip angle on the right side of the drawing body gradually increases.

As shown in Fig. 13b, with increasing seam dip angle, both $K_{1}$ and $f_{1}$ also show a gradually increasing trend. Furthermore, when the seam dip angle is $10^{\circ}$ or $20^{\circ}, f_{1}$ is negative. This indicates that owing to the rapid motion of particles in $\mathrm{DA}_{2}$, the direction of the frictional force on particles in $\mathrm{DA}_{1}$ is toward the point $O$, which makes the particles in $\mathrm{DA}_{1}$ begin to flow toward the opening, increasing the value of $m_{c}$ on the upper side of the opening. In contrast, when the seam dip angle is $30^{\circ}, 40^{\circ}$, or $50^{\circ}, f_{1}$ is positive. This is because at the beginning of the drawing process in a seam with a larger dip angle, the particles in $\mathrm{DA}_{1}$ cannot remain stationary, and the particle velocity is larger than that in $\mathrm{DA}_{2}$, so the direction of the frictional force on particles in $\mathrm{DA}_{1}$ is away from the point $O$. In addition, $K_{1}$ increases with increasing seam dip angle, and the size of the variation development area also increases accordingly. This is why the shape asymmetry of the two sides of the drawing body becomes very great when the seam dip angle is $50^{\circ}$, as shown in Fig. 12c.

\subsubsection{Normal drawing stage}

Based on numerical simulations of top coal drawing for different seam dip angles under the same boundary of the top coal, the coordinates of the point $A$ can be determined by analyzing the initial coordinates of the first drawn rock particle. The equation for the drawing body in the normal drawing stage can then be obtained together with the related parameters, as shown in Table 2. Figure 14 compares the theoretical drawing body shapes in the normal drawing stage with those obtained from the numerical simulation. It can be seen that there is a good match, which again shows the validity of the theoretical equation. In addition, it can be seen that the variation development area of the drawing body grows larger and larger with increasing seam dip angle.

\subsubsection{Variations of $\theta_{A}$ and $\rho_{\max }$}

The variations of $\theta_{A}$ and $\rho_{\max }$ with seam dip angle in the initial and normal drawing stages are shown in Fig. 15. In the normal drawing stage, in contrast to the initial drawing stage, with increasing seam dip angle, $\theta_{A}$ first decreases and then increases under the influence of the boundary of top coal. However, $\theta_{A}$ is always greater in the normal drawing stage than in the initial drawing stage, which

Table 1 Drawing body parameters for different seam dip angles in the initial drawing stage

\begin{tabular}{llllcrrrr}
\hline Dip angles $\left(^{\circ}\right)$ & $\theta_{G}^{\prime}\left({ }^{\circ}\right)$ & $L_{O}(\mathrm{~m})$ & $L_{C}(\mathrm{~m})$ & $\theta_{A}\left(^{\circ}\right)$ & $\rho\left(\theta_{A}\right)(\mathrm{m})$ & $\rho_{\max }(\mathrm{m})$ & $K_{1}$ & $f_{1}$ \\
\hline 0 & 50.00 & 0.89 & 9.16 & 90.00 & 9.16 & 9.16 & 0 \\
10 & 57.09 & 1.01 & 9.40 & 91.90 & 9.35 & 9.37 & 0.023 & -0.890 \\
20 & 62.49 & 1.10 & 9.89 & 93.89 & 9.67 & 9.77 & 0.072 & -0.278 \\
30 & 66.92 & 1.15 & 10.69 & 95.96 & 10.14 & 10.38 & 0.142 \\
40 & 70.79 & 1.17 & 11.95 & 97.99 & 10.80 & 11.27 & 0.223 \\
50 & 74.38 & 1.15 & 14.01 & 100.40 & 11.68 & 12.57 & 0.278 \\
\hline
\end{tabular}



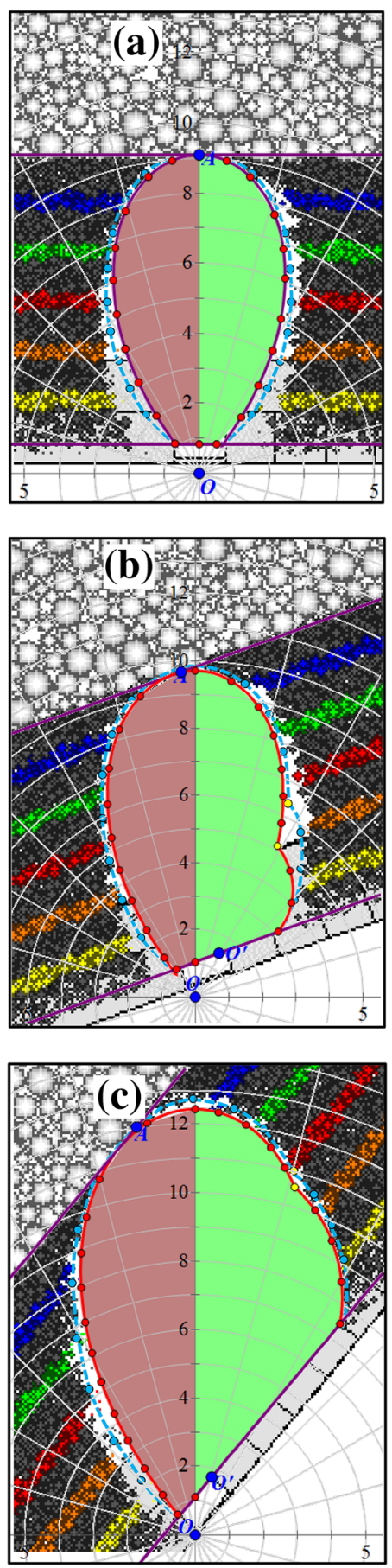

Fig. 12 Comparison of theoretical (red full curve) and numerically simulated (blue dashed curve) drawing body shapes in the initial drawing stage: $\mathbf{a} \alpha=0^{\circ} ; \mathbf{b} \alpha=20^{\circ} ; \mathbf{c} \alpha=50^{\circ}$

indicates that the position of the point $A$ on the left side of the drawing body is lower in the normal drawing stage. Moreover, with increasing seam dip angle, $\rho_{\max }$ in both the initial and normal drawing stages increases gradually, but the difference in $\rho_{\max }$ between the two stage decreases gradually, which shows that the constraint imposed by the
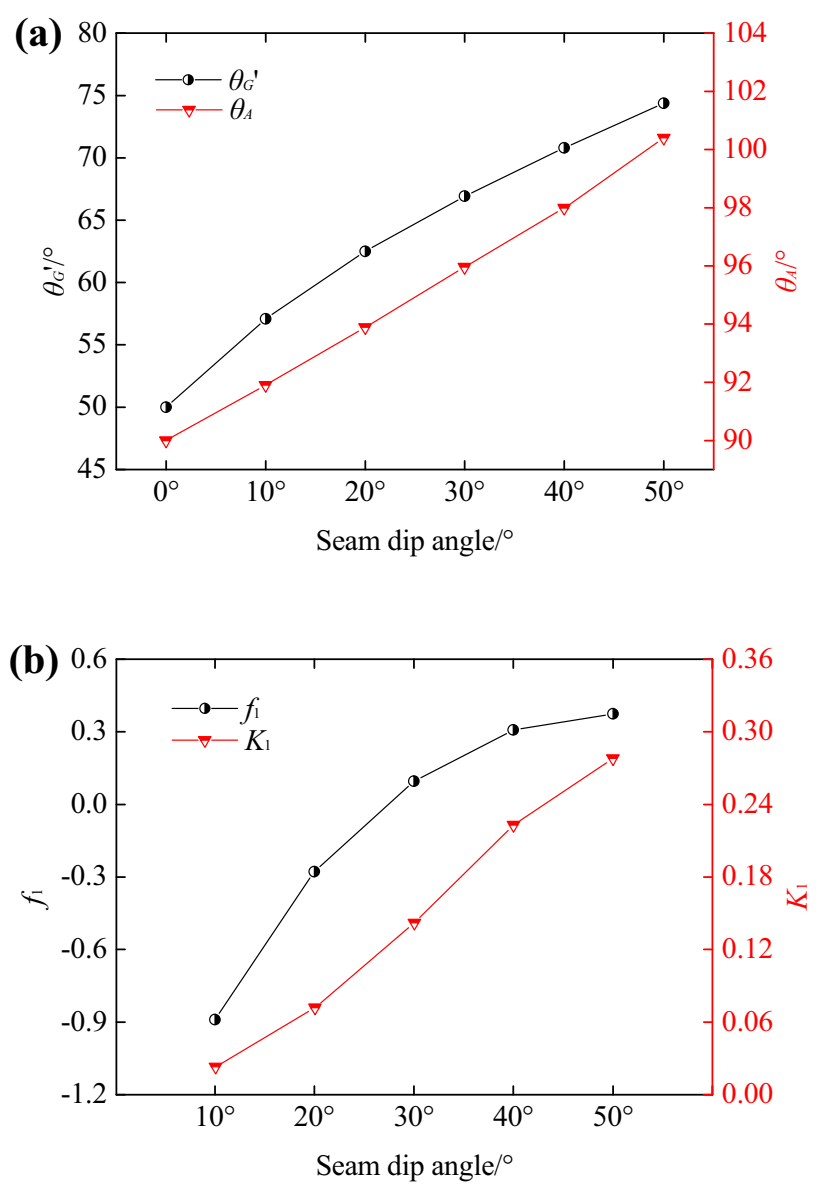

Fig. 13 Variations of drawing body parameters with increasing seam dip angle in the initial drawing stage: a $\theta_{G}^{\prime}$ and $\theta_{A} ; \mathbf{b} K_{1}$ and $f_{1}$

Table 2 Drawing body parameters for different seam dip angles in the normal drawing stage

\begin{tabular}{lllllll}
\hline $\begin{array}{l}\text { Dip angles } \\
\left({ }^{\circ}\right)\end{array}$ & $\begin{array}{l}\theta_{G}^{\prime} \\
\left(^{\circ}\right)\end{array}$ & $\theta_{A}\left({ }^{\circ}\right)$ & $\begin{array}{l}\rho\left(\theta_{A}\right) \\
(\mathrm{m})\end{array}$ & $\begin{array}{l}\rho_{\max } \\
(\mathrm{m})\end{array}$ & $K_{2}$ & $f_{2}$ \\
\hline 0 & 50.02 & 103.54 & 5.27 & 5.98 & 0 & - \\
10 & 57.09 & 103.28 & 6.53 & 7.37 & 0.024 & -0.419 \\
20 & 62.49 & 103.11 & 7.67 & 8.63 & 0.066 & -0.316 \\
30 & 66.92 & 108.57 & 7.84 & 10.08 & 0.141 & 0.099 \\
40 & 70.79 & 108.97 & 8.46 & 11.02 & 0.215 & 0.287 \\
50 & 74.38 & 109.55 & 9.41 & 12.47 & 0.290 & 0.399 \\
\hline
\end{tabular}

boundary of the top coal on the development of the drawing body decreases gradually. When the seam dip angle is larger than $30^{\circ}$, the difference in $\rho_{\max }$ is very small, and the constraint imposed by the boundary can be neglected. 

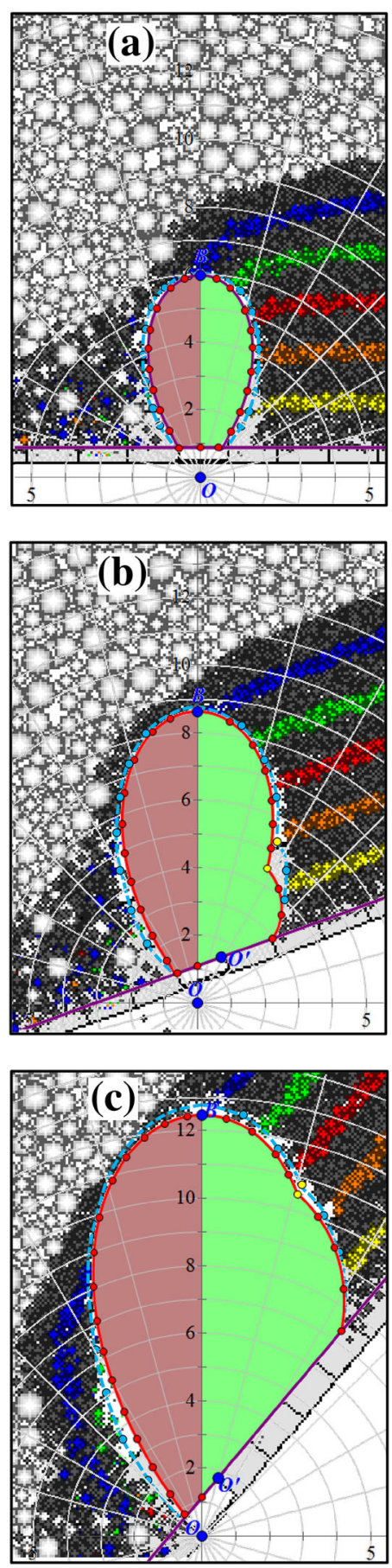

Fig. 14 Comparison of theoretical (red full curve) and numerically simulated (blue dashed curve) drawing body shapes in the normal drawing stage: $\mathbf{a} \alpha=0^{\circ} ; \mathbf{b} \alpha=20^{\circ} ; \mathbf{c} \alpha=50^{\circ}$

\subsection{Volume symmetry of the drawing body}

As shown in Fig. 16, we denote the volume of the left side of the drawing body in an inclined seam by $A_{l}$, the volume of the right side by $A_{r}$, and the total volume by $V$. In addition, we denote by $A_{1}$ the volume of the drawing body
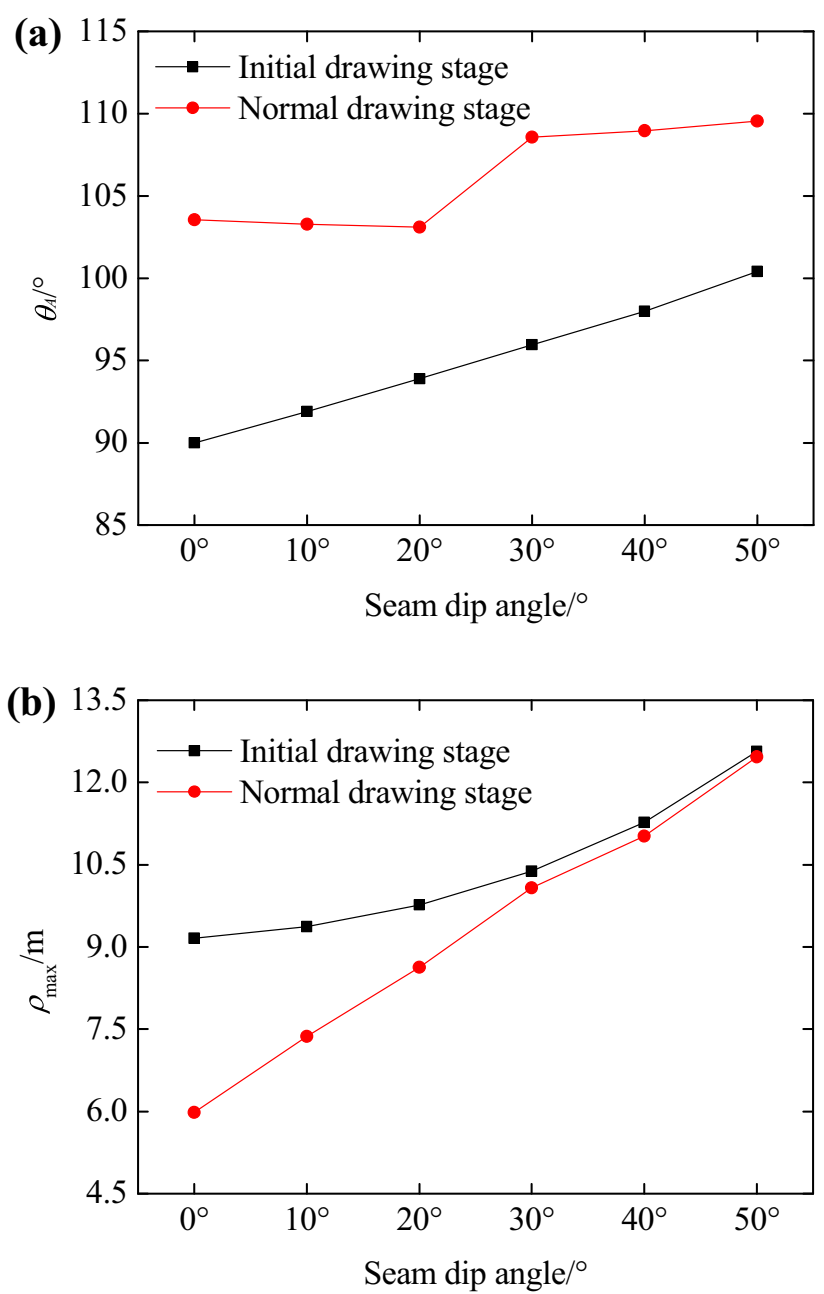

Fig. 15 Variations of $\mathbf{a} \theta_{A}$ and $\mathbf{b} \rho_{\max }$ in the initial and normal drawing stages

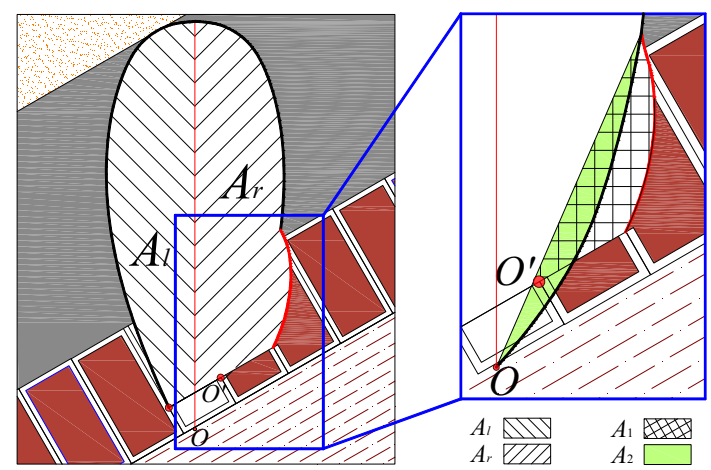

Fig. 16 Volume calculation for the drawing body in an inclined seam

in the angular range from $\alpha$ to $\theta_{G}^{\prime}$ in the new polar coordinates (the variation development volume) and by $A_{2}$ the volume of the drawing body in the angular range from $\theta_{G}$ to $\theta_{G}^{\prime}$ in the initial polar coordinates (the cut volume).

According to the B-R model, the volume of the drawing body in a flat seam, $A_{0}$, is given by 


$$
\begin{aligned}
A_{0}= & \int_{A} d A=\left(\frac{\rho_{\max }}{1-\sin \theta_{G}}\right)^{2} \int_{\theta_{G}}^{\pi / 2}\left(\sin \theta-\sin \theta_{G}\right)^{2} d \theta \\
= & \left(\frac{\rho_{\max }}{1-\sin \theta_{G}}\right)^{2}\left[\left(\frac{\pi}{2}-\theta_{G} \frac{\pi}{180}\right)\left(\sin ^{2} \theta_{G}+\frac{1}{2}\right)\right. \\
& \left.\quad-\frac{3}{2} \sin \theta_{G} \cos \theta_{G}\right]
\end{aligned}
$$

Therefore, $A_{l}$ can be calculated approximately as

$A_{l}=\frac{1}{2}\left(A_{0}-L_{O} \frac{L_{O W}}{2} \cos \alpha\right)$

The volume $A_{r}$ is given by

$$
\begin{aligned}
A_{r}=\frac{1}{2} & \left(\frac{\rho_{\max }}{1-\sin \theta_{G}}\right)^{2} \int_{\theta_{G}^{\prime}}^{\pi / 2}\left(\sin \theta-\sin \theta_{G}\right)^{2} d \theta \\
& -\frac{1}{2} L_{O} \frac{L_{O W}}{2} \cos \alpha \\
& +\frac{1}{2}\left(\frac{K_{1} \rho_{\max }}{1-\sin \theta_{G}}\right)^{2} \int_{\alpha}^{\theta_{G}^{\prime}}\left(\sin \theta-f_{1}\right)^{2} d \theta
\end{aligned}
$$

To study the effect of seam dip angle on the volume symmetry of the drawing body, the volume symmetry coefficient $\eta$ is defined as follows:

$$
\begin{aligned}
\eta= & \frac{A_{r}}{A_{l}}=1+\left(\frac{\rho_{\max }}{1-\sin \theta_{G}}\right)^{2} \\
& \times \frac{K_{1}^{2} \int_{\alpha}^{\theta_{G}^{\prime}}\left(\sin \theta-f_{1}\right)^{2} d \theta-\int_{\theta_{G}}^{\theta_{G}^{\prime}}\left(\sin \theta-\sin \theta_{G}\right)^{2} d \theta}{A_{0}-L_{O} \frac{L_{O W}}{2} \cos \alpha} \\
= & 1+\frac{A_{1}-A_{2}}{A}
\end{aligned}
$$

In the initial drawing stage, as shown in Table 3, the values of $A_{l}, A_{r}$, and $\eta$ for different seam dip angles can be obtained by inserting the parameters from Table 1 into Eqs. (26)-(28). From Fig. 17, it can be seen that with increasing seam dip angle, the total volume of the drawing body (the black plus red columns in Fig. 17) increases first

Table 3 Calculated drawing body volumes for different seam dip angles and volume symmetry coefficient in the initial drawing stage

\begin{tabular}{lllll}
\hline dip angles $\left(^{\circ}\right)$ & $A_{l}\left(\mathrm{~m}^{3}\right)$ & $A_{r}\left(\mathrm{~m}^{3}\right)$ & $A\left(\mathrm{~m}^{3}\right)$ & $\eta$ \\
\hline 0 & 14.92 & 14.92 & 29.84 & 1.000 \\
10 & 15.68 & 16.13 & 31.81 & 1.029 \\
20 & 17.02 & 18.92 & 35.94 & 1.112 \\
30 & 19.28 & 21.95 & 41.23 & 1.138 \\
40 & 22.80 & 25.68 & 48.48 & 1.126 \\
50 & 28.61 & 31.11 & 59.72 & 1.087 \\
\hline
\end{tabular}

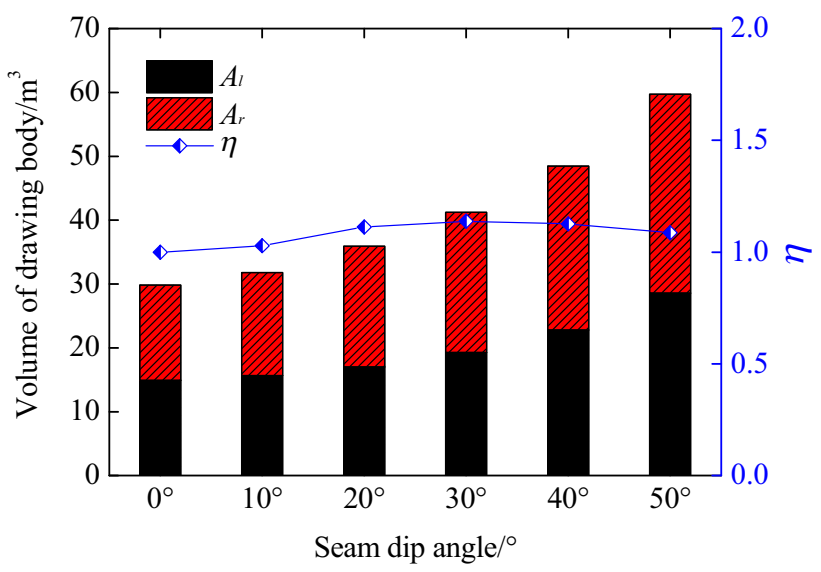

Fig. 17 Variations of drawing body volume and volume symmetry coefficient with seam dip angle

slowly and then rapidly. However, the value of $\eta$ always fluctuates around 1 as the seam dip angle varies. Thus, in the initial drawing stage, the shape of the drawing body is asymmetrical, although the volumes of its left and right sides are more or less the same.

In the normal drawing stage, similarly inserting the parameters from Table 2 into Eqs. (26)-(28) gives the values of $A_{l}, A_{r}$, and $\eta$ for different seam dip angles, as shown in Table 4.

The volume symmetry coefficients from theoretical calculation, numerical simulation, and physical experiments are shown in Fig. 18. The drawing body shape cannot be inverted in the normal drawing stage in physical drawing experiments, so there are no experimental results for $\eta$ in Fig. 18b. Both Fig. 18a and b indicate that with increasing seam dip angle, the value of $\eta$ fluctuates around 1. On the one hand, this verifies the correctness of the theoretical derivation. On the other hand, it illustrates that the drawing body exhibits the shape-difference and volume-symmetry characteristic in an inclined seam.

Table 4 Calculating drawing body volumes and volume symmetry coefficient for different seam dip angles in the normal drawing stage

\begin{tabular}{lrrrl}
\hline dip angles $\left(^{\circ}\right)$ & $A_{l}\left(\mathrm{~m}^{3}\right)$ & $A_{r}\left(\mathrm{~m}^{3}\right)$ & $A\left(\mathrm{~m}^{3}\right)$ & $\eta$ \\
\hline 0 & 6.20 & 6.20 & 12.40 & 1.000 \\
10 & 9.54 & 9.63 & 19.17 & 1.009 \\
20 & 13.18 & 14.46 & 27.64 & 1.097 \\
30 & 18.16 & 20.64 & 38.80 & 1.137 \\
40 & 21.82 & 24.57 & 46.39 & 1.126 \\
50 & 28.17 & 30.43 & 58.60 & 1.080 \\
\hline
\end{tabular}



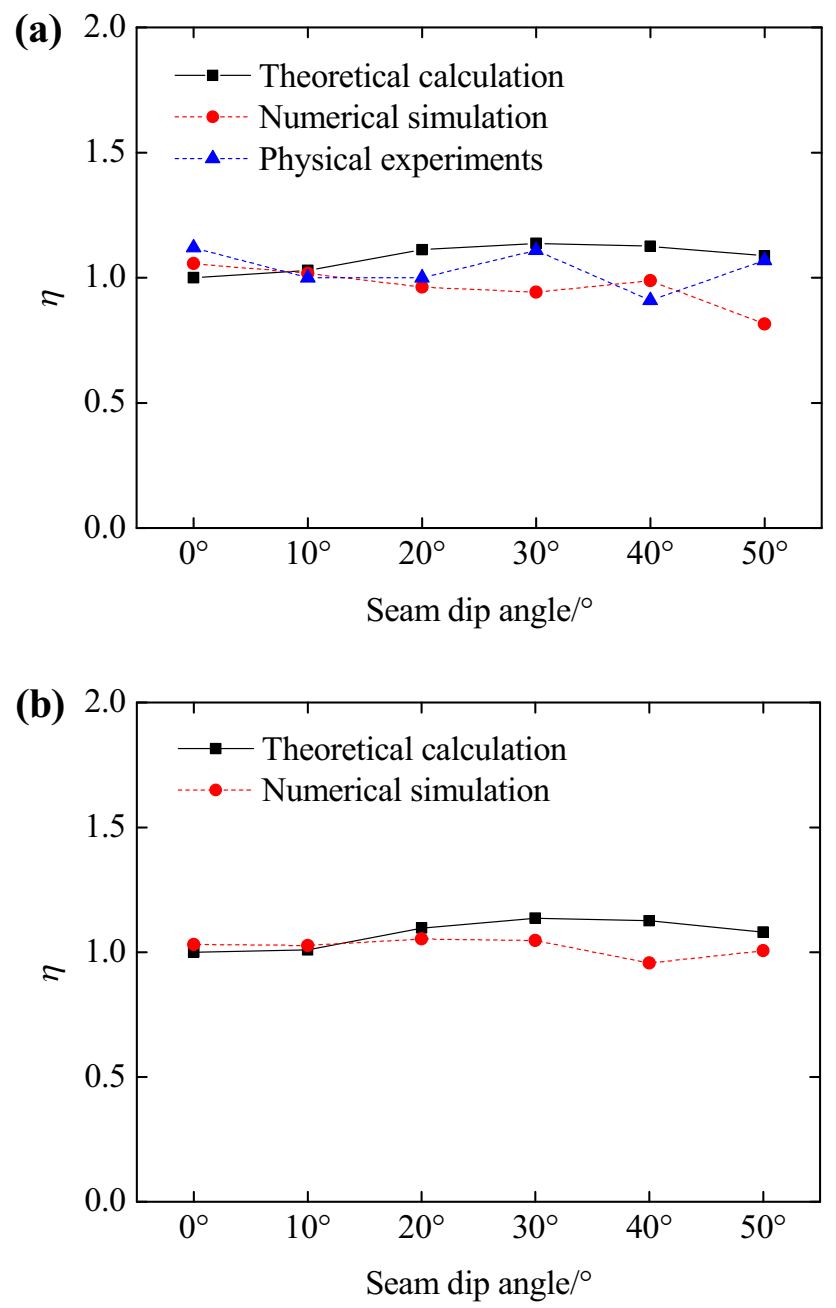

Fig. 18 Volume symmetry coefficients obtained from theoretical calculation, numerical simulation, and physical experiments: a initial drawing stage; b normal drawing stage

\section{Conclusions}

(1) Taking account of the influence of LTCC support and seam dip angle, theoretical models of the drawing body in the initial drawing stage and the normal drawing stage in an inclined seam have been established. A theoretical equation for the drawing body in an inclined seam has been proposed, which can accurately describe the characteristics of the drawing body shape.

(2) In an inclined seam, the shapes of the drawing body on the two sides of the axis of symmetry are clearly different. With increasing seam dip angle, the friction acting on the top coal particles gradually changes from negative to positive in the tracevariation zone, and the tendency of top coal particles to be drawn toward the upper side of the panel becomes more and more obvious. The size of the variation development area increases accordingly, which results in an asymmetry of the drawing body shape.

(3) The drawing body exhibits a shape-difference and volume-symmetry characteristic in an inclined seam. That is, with increasing seam dip angle, the shape asymmetry between the two sides of the drawing body becomes more and more obvious, although the volumes of the two sides remain nearly the same. This is because the variation development volume is more or less equal to the cut volume. Both theoretical calculation and numerical simulation confirm this shape-difference and volume-symmetry characteristic.

(4) The results of this study are of great significance for determining the drawing parameters and improving top coal recovery in inclined seams, especially coal seams with large dip angle.

Acknowledgements The authors gratefully acknowledge financial support from the Natural Science Foundation of China (51674264, 51574244), the National Key R\&D Plan of China (2018YFC0604501), and the China Postdoctoral Science Foundation (2018M631622). Special acknowledgements are also given to the China Scholarship Council (CSC).

\section{Compliance with ethical standards}

Conflict of interest All authors declare that they have no conflict of interest or financial conflicts to disclose.

Open Access This article is licensed under a Creative Commons Attribution 4.0 International License, which permits use, sharing, adaptation, distribution and reproduction in any medium or format, as long as you give appropriate credit to the original author(s) and the source, provide a link to the Creative Commons licence, and indicate if changes were made. The images or other third party material in this article are included in the article's Creative Commons licence, unless indicated otherwise in a credit line to the material. If material is not included in the article's Creative Commons licence and your intended use is not permitted by statutory regulation or exceeds the permitted use, you will need to obtain permission directly from the copyright holder. To view a copy of this licence, visit http://creativecommons. org/licenses/by/4.0/.

\section{References}

Jeromel G, Medved M, Likar J (2010) An analysis of the geomechanical processes in coal mining using the Velenje mining method. Acta Geotech Slov 7(1):30-45

Kumar R, Singh AK, Mishra AK, Singh R (2015) Underground mining of thick coal seams. Int J Min Sci Technol 25(6):885-896

Le TD, Oh J, Hebblewhite B, Zhang CG, Mitra R (2018) A discontinuum modelling approach for investigation of longwall top coal caving mechanisms. Int $\mathrm{J}$ Rock Mech Min Sci 106:84-95

Le TD, Zhang CG, Oh J, Mitra R, Hebblewhite B (2019) A new cavability assessment for longwall top coal caving from 
discontinuum numerical analysis. Int J Rock Mech Min Sci $115: 11-20$

Li XM, Wang ZH, Zhang JW (2017) Stability of roof structure and its control in steeply inclined coal seams. Int J Min Sci Technol 27(2):359-364

Likar J, Medved M, Lenart M, Mayer J, Malenković V, Jeromel G, Dervarič E (2012) Analysis of geomechanical changes in hanging wall caused by longwall multi top caving in coal mining. J Min Sci 48(1):135-145

Melo F, Vivanco F, Fuentes C et al (2007) On draw body shapes: from Bergmark-Roos to kinematic models. Int J Rock Mech Min Sci 44(1):77-86

Melo F, Vivanco F, Fuentes C (2009) Calculated isolated extracted and movement zones compared to scaled models for block caving. Int J Rock Mech Min Sci 46(4):731-737

Song Z, Konietzky H (2019) A particle-based numerical investigation on longwall top coal caving mining. Arabian J Geosci 12:556

Unver B, Yasitli NE (2006) Modelling of strata movement with a special reference to caving mechanism in thick seam coal mining. Int J Coal Geol 66(4):227-252

Vakili A, Hebblewhite BK (2010) A new cavability assessment criterion for longwall top-coal caving. Int J Rock Mech Min Sci 47(8):1317-1329

Verma P, Mishra B (2015) An experimental investigation of the creep behavior of an underground coal mine roof with shale formation. Int J Coal Geol 137(1):55-65
Wang JC, Zhang JW (2015) BBR study of top-coal drawing law in longwall top-coal caving mining. J China Coal Soc 40(3):487-493

Wang JC, Song ZY, Zhang JW et al (2016) Theoretical model of drawing body in LTCC mining. J China Coal Soc 41(2):352-358

Wang JC, Wei WJ, Zhang JW et al (2017) Stability analysis of support around the longwall top-coal caving mining in steeply thick coal seam. J China Coal Soc 42(11):2783-2791

Wang JC, Wei WJ, Zhang JW (2019) Effect of the size distribution of granular top coal on the drawing mechanism in LTCC. Granular Matter 21(3):70

Wei WJ, Song ZY, Zhang JW (2018) Theoretical equation of initial top-coal boundary in longwall top-coal caving mining. Int J Min Miner Eng 9(2):157-176

Yasitli NE, Unver B (2005) 3D numerical modeling of longwall mining with top-coal caving. Int $\mathrm{J}$ Rock Mech Min Sci 42(2):219-235

Yu B, Zhu DJ, Chen ZH (2017) Top-coal drawing law of LTCC mining based on stochastic medium theory. J China Coal Soc 42(6): 1366-1371

Zhang JW, Wang JC, Wei WJ (2018a) Effect of face dip angle on the drawing mechanism in longwall top-coal caving mining. J China Univ Min Technol 47(4):805-814

Zhang JW, Wang JC, Wei WJ, Chen Y, Song ZY (2018b) Experimental and numerical investigation on coal drawing from thick steep seam with longwall top coal caving mining. Arabian J Geosci 11:96 